\title{
Seguimiento por espectroscopia infrarroja (FT-IR) de la copolimerización de TEOS (tetraetilortosilicato) y PDMS (polidimetilsiloxano) en presencia de tbt (tetrabutiltitanio)
}

\author{
L. TÉLLEZ (1), F. RUBIO (2), R. PEÑA-ALONSO (2), J. RUBIO (2) \\ (1) Departamento de Metalurgia y Minerales, ESIQIE-Instituto Politécnico Nacional, UPALM-Zacatenco, México. e-mail: icvt343@icv.csic.es \\ (2) Departamento de Química-Física de Superficies y Procesos. Instituto de Cerámica y Vidrio. CSIC. Campus Cantoblanco. 28049 Madrid.
}

\begin{abstract}
Se han preparado materiales híbridos por medio de reacciones de hidrólisis y condensación de alcóxidos de Si y Ti (TEOS y TBT, respectivamente) y de reacciones de copolimerización de éstos con polidimetilsiloxano (PDMS). Se han estudiado las citadas reacciones mediante espectroscopia FT-IR, desde el mismo comienzo hasta la obtención del material final. La hidrólisis del TEOS así como la autocondensación del os grupos $\mathrm{Si}-\mathrm{OH}$ generados tanto para formar cadenas entrecruzadas como lineales se han seguido mediante las bandas situadas a 880, 1180 y $1150 \mathrm{~cm}^{-1}$, respectivamente. La policondensación de dichos grupos con PDMS se ha seguido por la banda a $850 \mathrm{~cm}^{-1}$. Por otro lado, la hidrólisis del TBT y la autocondensación de grupos Ti-OH se han seguido por la banda situada a 1130 y por la variación de la absorbancia en la región espectral $770-510 \mathrm{~cm}^{-1}$, respectivamente. La formación de enlaces Si-O-Ti por condensación de grupos $\mathrm{Si}-\mathrm{OH}$ y Ti-OH se ha seguido mediante la banda a $936 \mathrm{~cm}^{-1}$. Los resultados obtenidos han mostrado que las reacciones de hidrólisis y policondensación dependen de la concentración de TBT en el medio de reacción. La formación de estructuras entrecruzadas de enlaces Si-O-Si se ve favorecida por el TBT, si bien por el contrario éste impide la reacción de copolimerización TEOS-PDMS. La autocondensación de grupos Si-OH y Ti-OH es muy rápida dando lugar a enlaces $\mathrm{Si}-\mathrm{O}-\mathrm{Si}$ y Ti-O-Ti, respectivamente. También se forman enlaces Si-O-Ti en los comienzos de la reacción, sin embargo éstos tienden a desaparecer en el transcurso de la misma. Finalmente se ha estudiado la variación de la concentración de cadenas cíclicas y lineales de PDMS durante toda la reacción.
\end{abstract}

Palabras Clave: Ormosil, sol-gel, hidrólisis, condensación, FT-IR.

\section{FT-IR spectroscopy study of the reaction of obtention of hybrid materials}

Hybrid materials have been prepared in this work through the reactions of Si and Ti alkoxides (TEOS and TBT, respectively) and polydimethil siloxane (PDMS). These reactions have been studied by means of FT-IR spectroscopy during the whole reaction time. The hydrolysis of TEOS molecule has been followed by the $880 \mathrm{~cm}^{-1}$ band, and the self-condensation reactions through the 1180 and $1150 \mathrm{~cm}^{-1}$ bands. Polycondesation reaction between Si-OH groups and PDMS molecules has been followed by the $850 \mathrm{~cm}^{-1}$ band. On the other hand, the hydrolysis reaction of TBT and the self-condensation of Ti-OH groups have been followed by the 1130 and $770-510 \mathrm{~cm}^{-1}$ bands, respectively. Finally the condensation reaction between $\mathrm{Si}-\mathrm{OH}$ and Ti-OH groups have been studied by the $936 \mathrm{~cm}^{-1}$ band. Results have shown that hydrolysis and condensation reactions are depending on TBT concentration. The formation of Si-O-Si cross-linked structures increases with the TBT concentrations in the reaction. The selfcondensation reaction of $\mathrm{Si}-\mathrm{OH}$ grups or $\mathrm{Ti}-\mathrm{OH}$ grous is very reapid forming $\mathrm{Si}-\mathrm{O}-\mathrm{Si}$ and $\mathrm{Ti}-\mathrm{O}-\mathrm{Ti}$ bonds, respectively. However, the $\mathrm{Si}-\mathrm{O}-\mathrm{Ti}$ bonds which are formed during the first moments of reaction are also rapidly broken due to $\mathrm{H}_{2} \mathrm{O}$ molecules or the reaction medium. The evolution of PDMS linear and cyclic molecules is also studied.

Key Words: Ormosil, sol-gel, hydrolysis, condensation, FT-IR.

\section{INTRODUCCIÓN}

Es bien conocido que el método sol-gel es un proceso que permite preparar materiales vítreos y cerámicos con una serie de ventajas como son las bajas temperaturas (relativas) de procesado, la posibilidad de obtener materiales muy variados tanto en composición como en forma, la elevada pureza de los materiales, etc $(1,2)$. Así, se han preparado distintos materiales vítreos con diferentes composiciones, vitrocerámicos, cerámicos tales como $\mathrm{Al}_{2} \mathrm{O}_{3^{\prime}}, \mathrm{TiO}_{2^{\prime}}$ mullita, etc., así como fibras, capas y recubrimientos, piezas monolíticas y/o porosas, etc (1).

Por otro lado, desde hace varios años el método sol-gel también se está utilizando para sintetizar materiales híbridos orgánico-inorgánicos $(3,4)$. Las reacciones involucradas en esta síntesis son las mismas que las que rigen el proceso solgel para la obtención de materiales inorgánicos puros $(5,6)$. En este sentido, la obtención de materiales híbridos se lleva a cabo incorporando polímeros orgánicos en la propia red inorgánica. Los materiales así obtenidos se les conoce con el nombre de Ormosiles, Ceramers o Polycerams. La característica más importante de estos materiales híbridos se basa en que las moléculas orgánicas deben estar lo más dispersas posibles en todo el material, consiguiéndose así una distribución homogénea a nivel molecular.

Las materias primas que se utilizan de forma fundamental para la obtención de materiales híbridos son los alcóxidos de silicio, en los que alguno de los radicales está substituido por uno no hidrolizable (7). El alcóxido más utilizado es el TEOS (tetraetil ortosilicato), ya que posee una velocidad de hidrólisis que puede ser controlada, a la vez que permite su copolimerización con otros alcóxidos alquil substituidos de silicio $(8,9)$. 
Muchos trabajos han demostrado que es posible utilizar polímeros orgánicos para la obtención de materiales híbridos $(10,11)$. Para ello se debe poseer un polímero cuyas terminaciones sean grupos silanol. De esta forma estos polímeros pueden reaccionar no solo con el TEOS hidrolizado (con los grupos Si-OH), sino también con los grupos hidroxilo de otros alcóxidos tales como los de titanio, aluminio, zirconio, etc. (12-14).

Para el estudio de las reacciones de obtención de materiales híbridos se han utilizado numerosas técnicas experimentales, encontrándose entre ellas las espectroscopias infrarroja, Raman y resonancia magnética nuclear (RMN), etc. (13-16). Así las reacciones entre TEOS-PDMS o TEOS-DMDES (dimetil-dietoxisilano) y alcóxidos de titanio se ha estudiado con gran profundidad mediante ${ }^{29} \mathrm{Si}$ RMN (17). Por otro lado, la evolución estructural así como las propiedades de estos materiales se han seguido mediante ${ }^{17} \mathrm{O} R M N,{ }^{1} \mathrm{H}$ RMN y espectroscopia de absorción de rayos $X(6,12,17-19)$. Sin embargo la espectroscopia infrarroja ha sido muy poco utilizada hasta la fecha. Es por ello por lo que en este trabajo se ha utilizado esta espectroscopia para seguir las reacciones de hidrólisis y policondensación que tienen lugar en la preparación de materiales híbridos a base de TEOS, PDMS y TBT. Este sistema ya ha sido investigado por otros autores mediante espectroscopia RMN $(6,17)$, si bien en el trabajo que aquí se presenta podrá comprobarse como mediante FT-IR es posible realizar un mayor número de espectros y llegar a conclusiones similares y utilizando un equipo bastante más accesible.

\section{EXPERIMENTAL}

\subsection{Materiales}

Los materiales híbridos se prepararon utilizando TEOS (Merck p.a.), polidimetilsiloxano terminado en hidroxilos (PDMS) de peso molecular 550 g.mol ${ }^{-1}$ (Gelest), TBT (Aldrich, p.a.), isopropanol $(\mathrm{iPrOH})$, ácido clorhídrico $(\mathrm{HCl})$ y agua destilada-desionizada $\left(\mathrm{H}_{2} \mathrm{O}\right)$. Estos materiales fueron obtenidos con una relación en peso constante inorgánico/orgánico de 70/30, donde la parte inorgánica se considera que es la suma de TEOS y TBT, y la parte orgánica lo forma solamente el PDMS. Con respecto a la parte inorgánica, ésta se varió de acuerdo con TEOS $/$ TBT $=70 / 0,69 / 1,65 / 5$ y 63/7, respectivamente. Por otro lado, se mantuvieron constantes las relaciones molares siguientes: $\mathrm{HCl}$ / Inorgánico $=0.1, \mathrm{H}_{2} \mathrm{O} /$ Inorgánico = 3, e iPrOH/Inorgánico $=4.5$.

La forma de obtención de estos materiales fue la siguiente: se prepararon tres soluciones, una de ellas contenía los volúmenes totales de TEOS y de PDMS y la tercera parte del volumen de $\mathrm{iPrOH}$, otra disolución contenía los volúmenes totales de $\mathrm{H}_{2} \mathrm{O}$ y $\mathrm{HCl}$ así como otra tercera parte del volumen de $\mathrm{iPrOH}$, y la última disolución contenía el volumen total de TBT y la tercera parte del volumen de iPrOH. Las tres soluciones se mantuvieron en agitación durante dos horas a 80 ${ }^{\circ} \mathrm{C}$. Transcurrido este tiempo la segunda disolución se añadió directamente sobre la primera de forma rápida. Así se obtuvo una disolución homogénea sobre la cual inmediatamente a continuación la tercera disolución se fue añadiendo gota a gota durante un periodo de 35 minutos. De esta forma se consiguió que el TBT añadido no precipitara, siendo la disolución final completamente homogénea. Esta disolución final se mantuvo a la temperatura indicada durante un tiempo adicional de 25 minutos. Durante todo este tiempo se mantuvo una agitación constante con reflujo en el matraz donde se llevó a cabo la reacción. Transcurrido este tiempo de 60 minutos, cada disolución se depositó sobre un recipiente de plástico, sellándose éste hasta que se observó que las muestras habían gelificado. El tiempo de gelificación fue de 44, 47, 49, 54 y 59 horas para las muestras que contenían el 0, 1, 3, 5 y $7 \%$ de TBT, respectivamente. Todas las muestras gelificadas eran completamente transparentes y monolíticas, no observándose ni precipitados ni separación de fases en ellas.

\subsection{Métodos.}

El estudio de las reacciones de hidrólisis y policondensación de los sistemas anteriores se llevó a cabo utilizando un espectrofotómetro de infrarrojo (FT-IR) Perkin-Elmer 1720 X, con una resolución de $2 \mathrm{~cm}^{-1}$. Cada espectro obtenido es la media de 10 medidas realizadas sobre la misma muestra la cual consta de un micro-gota de $1 \mu$ l añadida entre dos ventanas de cristales de KRS-5 (bromuro-ioduro de talio) también de Perkin-Elmer, cristales transparentes al infrarrojo. Para este proceso se utilizó una micro-pipeta calibrada previamente, y además se utilizó una punta distinta para cada medida con el fin de evitar contaminaciones. El tiempo transcurrido entre la primera y la décima medida es de unos 30 segundos, por lo que puede considerarse que durante este tiempo la reacción prácticamente no transcurre (si se compara con las más de 40 horas necesarias para la gelificación, tal y como se ha comentado anteriormente) y, por ello, el espectro obtenido es una representación de la reacción en dicho momento de reacción. Los espectros FT-IR se realizaron desde el comienzo de la adición de las dos primeras soluciones hasta el momento en el cual se observó que la disolución había gelificado. Los espectros se guardaron en el ordenador hasta que fueron analizados. Durante el periodo de reacción de 60 minutos los espectros fueron tomados en intervalos de 2 minutos, mientras que a partir de ese tiempo el intervalo se fue ampliando hasta llegar a la gelificación.

\section{RESULTADOS}

Para la obtención de materiales híbridos a base de TEOS y PDMS se ha propuesto un mecanismo de reacción similar al del proceso sol-gel en el que se dan las reacciones de hidrólisis y policondensación entre los reactantes. El mecanismo primero propuesto fue el de Wilkes y col. (11), el cual solamente consideraba la reacción de hidrólisis del TEOS y las de policondensación del TEOS hidrolizado con el PDMS. Sin embargo más tarde Iwamoto y col. (14) propusieron un nuevo mecanismo en el cual además de dichas reacciones se tenían en cuenta la formación de tetrámeros cícliclos así como la rotura de las moléculas de PDMS en fragmentos más cortos. Este mecanismo de Iwamoto y col. (14) estaba de acuerdo con lo presentado por Glaser (15) el cual mostró que se podían formar tetrámeros cíclicos $\left(\mathrm{D}_{4}\right.$, octametilciclotetrasiloxano) a partir de moléculas de PDMS en un medio catalizado por ácidos. En resumen, el esquema de reacción de este sistema es el siguiente:

\section{Hidrólisis:}

$\mathrm{Si}-(\mathrm{OEt})_{4}+\mathrm{H}_{2} \mathrm{O} \rightarrow \mathrm{Si}-(\mathrm{OH})_{4}+4 \mathrm{EtOH}$ 
Donde OEt es el grupo etóxido que proporciona etanol (EtOH) una vez hidrolizado.

\section{Policondensación:}

\section{Autocondensación del TEOS:}

$\mathrm{Si}(\mathrm{OR})_{4}+\mathrm{Si}(\mathrm{OH})_{4} \rightarrow(\mathrm{OR})_{3} \mathrm{Si}-\mathrm{O}-\mathrm{Si}-(\mathrm{OH})_{3}+\mathrm{H}_{2} \mathrm{O}$

Copolimerización del TEOS con el PDMS:

$2 \mathrm{Si}(\mathrm{OH})_{4}+\mathrm{HO}-\mathrm{Si}\left(\mathrm{CH}_{3}\right)_{2}\left(-\mathrm{O}-\mathrm{Si}\left(\mathrm{CH}_{3}\right)_{2}\right)_{\mathrm{x}}-\mathrm{O}-\mathrm{Si}\left(\mathrm{CH}_{3}\right)_{2}-\mathrm{OH} \rightarrow$

$(\mathrm{HO})_{3}-\mathrm{Si}-\mathrm{O}\left(\mathrm{Si}\left(\mathrm{CH}_{3}\right)_{2}\left(-\mathrm{O}-\mathrm{Si}\left(\mathrm{CH}_{3}\right)_{2}\right)_{\mathrm{y}}-\mathrm{O}-\mathrm{Si}-(\mathrm{OH})_{3}+\right.$

$+(\mathrm{HO})_{3}-\mathrm{Si}-\mathrm{O}\left(\mathrm{Si}\left(\mathrm{CH}_{3}\right)_{2}\left(-\mathrm{O}-\mathrm{Si}\left(\mathrm{CH}_{3}\right)_{2}\right)_{\mathrm{z}}-\mathrm{O}-\mathrm{Si}-(\mathrm{OH})_{3}\right.$

+ ciclo- $\left(\mathrm{Si}\left(\mathrm{CH}_{3}\right)_{2}-\mathrm{O}-\right)_{4}+2 \mathrm{H}_{2} \mathrm{O} \quad(\mathrm{x}>\mathrm{y}, \mathrm{z})$

Como puede verse la reacción de copolimerización tiene lugar entre los hidroxilos libres (silanoles) de las estructuras autocondensadas del TEOS y los grupos hidroxilo de final de cadena del PDMS. A su vez se observa que las cadenas de PDMS pueden romperse dando lugar a cadenas más cortas y a tetrámeros ciclos de polisiloxano.

En el modelo propuesto anteriormente se considera solamente la reacción de hidrólisis del TEOS, si bien es cierto que otras reacciones son posibles tales como la olación y la oxolación. Es de decir que dada la elevada acidez del medio en el que se da la reacción las dos últimas reacciones están minimizadas.

La incorporación de TBT en este sistema de TEOS-PDMS no debe en principio alterar las dos reacciones anteriores [1] y [3], si bien como este alcóxido también se hidroliza se formarán grupos Ti-OH los cuales se autocondensarán para formar estructuras con enlaces Ti-O-Ti, a la vez que podrán policondensar tanto con TEOS (hidrolizado) como con PDMS dando lugar a la formación de enlaces Ti-O-Si. Ahora bien, teniendo en cuenta las moléculas de $\mathrm{H}_{2} \mathrm{O}$ que son necesarias para hidrolizar completamente al TEOS o al TBT, y que de acuerdo a como se ha llevado a cabo la reacción, en donde los 3 moles de $\mathrm{H}_{2} \mathrm{O}$ se añadían completamente al principio a la disolución que contenía el TEOS (es decir se llevaba a cabo una prehidrólisis), la reacción de hidrólisis del TBT estará condicionada a la presencia de moléculas de $\mathrm{H}_{2} \mathrm{O}$ en el medio de reacción, moléculas que se generarán a partir de las reacciones [2] y [3] anteriores. Pues bien, tal y como se comentó al principio, en este trabajo se ha utilizado la espectroscopia FT-IR para seguir estas reacciones desde el momento mismo de la adición de la disolución hidrolizante $\left(\mathrm{H}_{2} \mathrm{O} / \mathrm{HCl}\right)$ hasta el momento de la gelificación del sistema.

En la Figura 1 se muestran los espectros correspondientes a las materias primas de partida, y en la Tabla 1 se recogen las bandas fundamentales de absorción IR. En el espectro de la disolución mezcla de TEOS y PDMS se pueden diferenciar tres zonas espectrales: la comprendida entre 4000 y $2500 \mathrm{~cm}^{-1}$, la correspondiente a la región 1300-1000 $\mathrm{cm}^{-1}$, y la última comprendida entre 1000 y $400 \mathrm{~cm}^{-1}$. En la primera región espectral tienen lugar las frecuencias de vibración de tensión de los enlaces $\mathrm{O}-\mathrm{H}$ y $\mathrm{C}-\mathrm{H}$, y en la cual no nos vamos a detener. En este sentido el enlace $\mathrm{C}-\mathrm{H}$ tiene una vibración de flexión fuera del plano la cual da una banda intensa a $1375 \mathrm{~cm}^{-1}$ la cual aparece claramente en los espectros. En la segunda región espectral las bandas principales aparecen a $1262 \mathrm{~cm}^{-1}\left(\rho \mathrm{CH}_{3^{\prime}}\right.$ PDMS) y a $1168 \mathrm{~cm}^{-1}$ (rocking del $\mathrm{CH}_{3}$, TEOS). En esta misma zona las bandas que aparecen a 1104, 1084 y 1030 se deben tanto al TEOS como al PDMS (vSi-O) y al iPrOH (vC-O). En el espectro

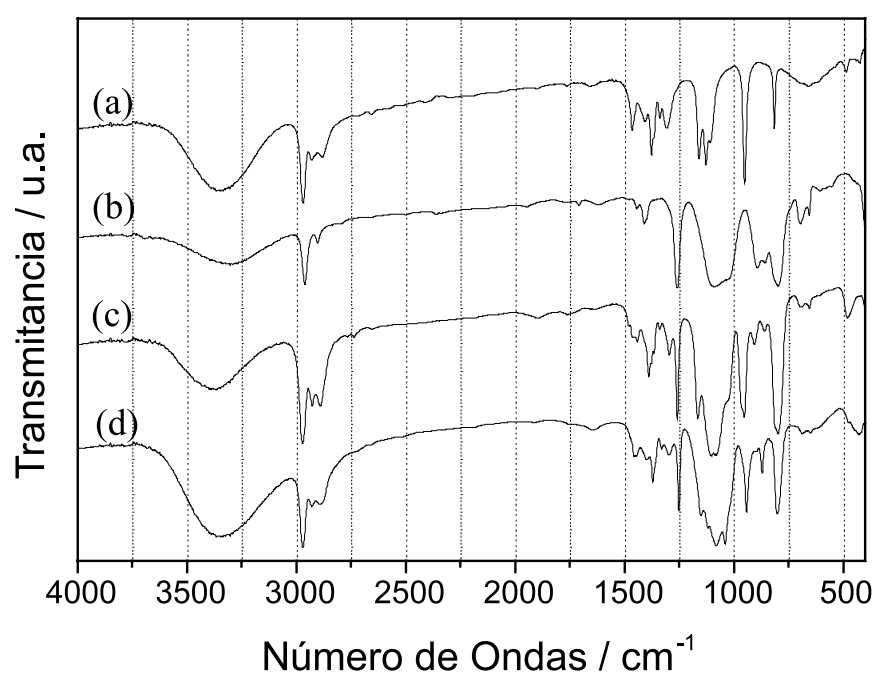

Figura 1. Espectros infrarrojos del TEOS, PDMS, de la disolución TEOS-PDMS, y de la disolución TEOS-PDMS inmediatamente después de ser añadida la disolución de hidrólisis.

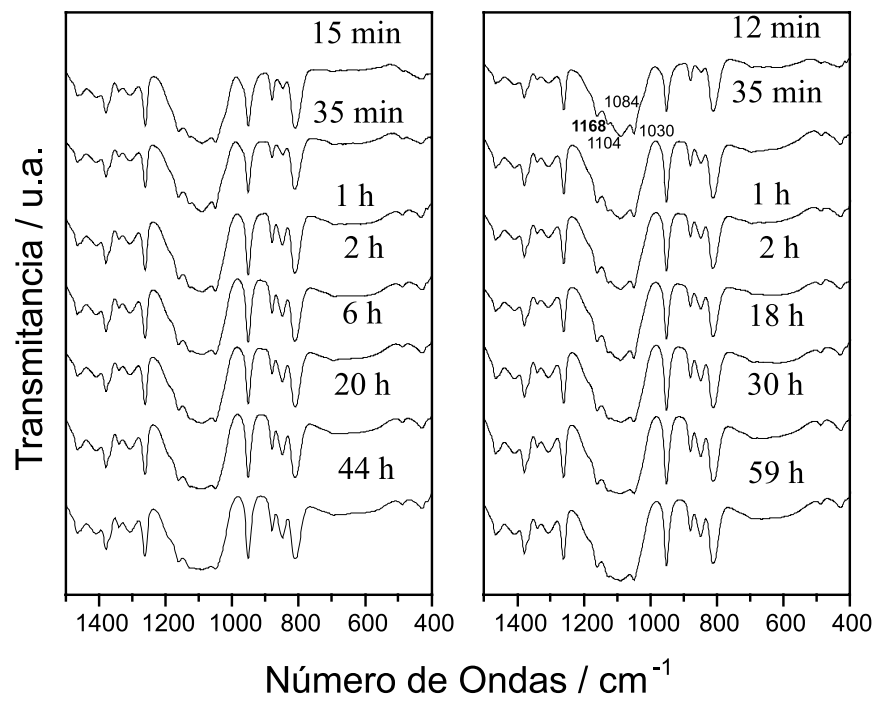

Figura 2. Espectros infrarrojos para diferentes tiempos de la reacción de obtención de ormosiles. (a) sin TBT, y (b) con 7\% de TBT.

de la Figura 2 correspondiente a los 12 minutos de reacción se han indicado estas bandas por medio de los números a los que aparecen. En la tercera y última zona espectral se pueden observar las bandas situadas a $953 \mathrm{~cm}^{-1}\left(\delta_{\mathrm{CH}_{3}}, \mathrm{iPrOH}\right)$ y 798 $\mathrm{cm}^{-1}$ (USi-O, TEOS y PDMS), así como dos pequeños hombros sobre los $966 \mathrm{~cm}^{-1}$ (rocking $\mathrm{CH}_{3^{\prime}}$ TEOS) y sobre los $817 \mathrm{~cm}^{-1}$ (rocking $\mathrm{CH}_{2}$, iPrOH). Aparecen también en esta zona dos bandas muy pequeñas a 909 y $862 \mathrm{~cm}^{-1}$ que son originadas por el PDMS (Figura 1b) y las cuales cambian en intensidad cuando el PDMS está diluido (Figura 1c), pues se rompen los enlaces de hidrógeno existentes entre las moléculas de PDMS en el proceso de dilución (20). En esta misma Figura 1 se muestra el espectro nada más ser añadida la disolución hidrolizante de $\mathrm{HCl}-\mathrm{H}_{2} \mathrm{O}$ (Figura 1d). Puede verse en este espectro como los cambios fundamentales ocurren en la región espectral 1200$800 \mathrm{~cm}^{-1}$, pues desaparecen las bandas fundamentales originadas por el TEOS $\left(1168,965\right.$ y $\left.798 \mathrm{~cm}^{-1}\right)$ a la vez que se forma etanol (banda situada a $881 \mathrm{~cm}^{-1}$ ) por hidrólisis del TEOS. 
TABLA 1. ASIGNACIÓN DE LAS BANDAS FUNDAMENTALES DE ABSORCIÓN IR DE LOS MATERIALES DE PARTIDA.

\begin{tabular}{|c|c|c|c|c|c|}
\hline \multicolumn{2}{|c|}{ PDMS } & \multicolumn{2}{|c|}{ TEOS } & \multicolumn{2}{|c|}{ TBT } \\
\hline $\begin{array}{l}\text { Posición } \\
\left(\mathrm{cm}^{-1}\right)\end{array}$ & Asignación & $\begin{array}{l}\text { Posición } \\
\left(\mathrm{cm}^{-1}\right)\end{array}$ & Asignación & $\begin{array}{l}\text { Posición } \\
\left(\mathrm{cm}^{-1}\right)\end{array}$ & Asignación \\
\hline 3306 & vO-H & 2976 & $\mathrm{v}_{\mathrm{ac} \cdot \mathrm{H}}$ en $\mathrm{CH}_{3}$ & 3330 & $\mathrm{vO}-\mathrm{H}$ \\
\hline 2964 & $\mathrm{v}_{\mathrm{aCH}}$ en $\mathrm{CH}_{3}$ & 2930 & $v_{\mathrm{ac} \cdot \mathrm{H}}$ en $\mathrm{CH}_{2}$ & 2960 & $v_{\mathrm{ac}-\mathrm{H}}$ en $\mathrm{CH}_{3}$ \\
\hline 2905 & $\mathrm{v}_{\mathrm{sC} \cdot \mathrm{H}}$ en $\mathrm{CH}_{3}$ & 2891 & $v_{\mathrm{sC}-\mathrm{H}}$ en $\mathrm{CH}_{3}$ & 2930 & $v_{\mathrm{aCCH}}$ en $\mathrm{CH}_{2}$ \\
\hline 1413 & $\mathrm{\delta}_{\mathrm{a}} \mathrm{CH}_{3}$ & 1484 & $\delta_{\mathrm{ac}-\mathrm{H}}$ en $\mathrm{CH}_{2}$ & 2870 & $\mathrm{v}_{\mathrm{ac}-\mathrm{H}}$ en $\mathrm{CH}_{3}$ \\
\hline 1262 & $\delta_{\mathrm{sCH} 3}$ en $\mathrm{Si}\left(\mathrm{CH}_{3}\right)_{2}$ & 1444 & $\delta_{\mathrm{sC} \cdot \mathrm{H}}$ en $\mathrm{CH}_{2}-\mathrm{O}$ & 1465 & $\delta_{\mathrm{aC}-\mathrm{H}}$ en $\mathrm{CH}_{2}$ \\
\hline 1088,1034 & $v_{\mathrm{a} \mathrm{si-O}}$ en Si-O-Si & 1391 & $\delta_{\mathrm{sC}-\mathrm{H}}$ en $\mathrm{CH}_{3}$ & 1375 & $\delta_{\mathrm{sC} \cdot \mathrm{H}}$ en $\mathrm{CH}_{3}$ \\
\hline 895 & $\mathrm{v}_{\mathrm{a} \text { si-O }}$ en Si-OH & 1279 & $\delta_{w c-H}$ en $\mathrm{CH}_{3}$ & 1125 & $v_{\text {Ti-OC }}$ en $\mathrm{CH}_{3}-\left(\mathrm{CH}_{2}\right)_{3}-\mathrm{O}$ \\
\hline 860 & $\mathrm{CH}_{3}$ rocking & 1170 & $\mathrm{CH}_{3}$ rocking & 1085 & $v_{\text {Ti.O. }}$ en $\mathrm{CH}_{3}-\left(\mathrm{CH}_{2}\right)_{3}-\mathrm{O}$ \\
\hline 805 & $v_{\mathrm{a}} \mathrm{Si}-\mathrm{C}$ & 1108 & $v_{\mathrm{a}} \mathrm{Si}-\mathrm{O}-\mathrm{C}$ & 1035 & $v_{\mathrm{Ti}-\mathrm{O} C}$ en $\mathrm{CH}_{3}-\left(\mathrm{CH}_{2}\right)_{3}-\mathrm{O}$ \\
\hline 698 & $\mathrm{v}_{\mathrm{S}} \mathrm{Si}-\mathrm{C}$ & 1080 & $v_{\mathrm{s}} \mathrm{Si}-\mathrm{O}-\mathrm{C}$ & 864 & v C-O \\
\hline 658 & $\mathrm{Si}-\mathrm{CH}_{3}$ rocking & 968 & $\mathrm{CH}_{3}$ rocking & 606 & $v$ Ti-O \\
\hline & & 810 & $\mathrm{CH}_{2}$ rocking & & \\
\hline & & 795 & $\mathrm{SiO}_{4}$ a. & & \\
\hline & & 656 & $\mathrm{SiO}_{4} \mathrm{~s}$. & & \\
\hline & & 480 & $\delta \mathrm{O}-\mathrm{C}-\mathrm{C}$ & & \\
\hline & & 464 & $\delta \mathrm{O}-\mathrm{Si}-\mathrm{O}$ & & \\
\hline
\end{tabular}

En la Figura 2 se muestran los espectros del transcurso de la reacción de obtención de materiales híbridos a base de TEOS y PDMS cuando no se adiciona TBT o bien cuado se adiciona el $7 \%$ de TBT a dicho medio (Figuras $2 \mathrm{a}$ y $2 \mathrm{~b}$ respectivamente). En esta Figura 2 y en el espectro correspondiente a los 12 minutos de reacción se muestran más claramente las bandas situadas a 1168, 1104, 1084 y $1030 \mathrm{~cm}^{-1}$, bandas que están en todos los espectros. El espectro final de esta Figura 2 corresponde a la muestra unos minutos antes de gelificar. Como puede verse los espectros de ambas muestras son muy similares pudiéndose identificar las siguientes diferencias en ellos: a) la autocondensación de los grupos Si-OH para formar enlaces Si-O-Si (hombro próximo a $1180 \mathrm{~cm}^{-1}$ ), b) la copolimerización de los grupos Si-OH del TEOS con las moléculas de PDMS (banda situada a $\left.850 \mathrm{~cm}^{-1}\right), \mathrm{y} \mathrm{c}$ ) un pequeño incremento del área de la zona espectral comprendida entre 770 y $500 \mathrm{~cm}^{-1}$ en la muestra que contiene el $7 \%$ de TBT, lo que indica la formación de enlaces Ti-O-Ti por autocondensación de grupos Ti-OH procedentes del TBT hidrolizado. Puede comprobarse también como la banda de etanol a $881 \mathrm{~cm}^{-1}$ permanece constante en todos los espectros de la Figura 2, lo que indica que desde los primeros momentos ya no se forma más etanol en el medio, por lo que puede decirse que el TEOS se hidroliza instantáneamente nada más se añadida la disolución hidrolizante.

La presencia de enlaces Ti-O-Ti se puede apreciar con bastante claridad en la Figura 3 en la que se presentan los espectros de las muestras con distintas concentraciones de TBT justo unos momentos antes de que estas gelifiquen. Puede verse como la región espectral $770-500 \mathrm{~cm}^{-1}$ aumenta en intensidad con la concentración de TBT en el medio lo que indica que se están formado los citados enlaces y que corresponden a una estructura amorfa de hidróxido de titanio hidratado $(21,22)$.

\section{DISCUSIÓN}

Con el fin de obtener más información de los espectros cualitativos que se acaban de exponer, se ha procedido a realizar la deconvolución de cada espectro en el intervalo 1260-400 $\mathrm{cm}^{-1}$, para ello se ha asumido que las bandas IR que lo forman son todas de tipo gauss. La reacción de hidrólisis de la molé-

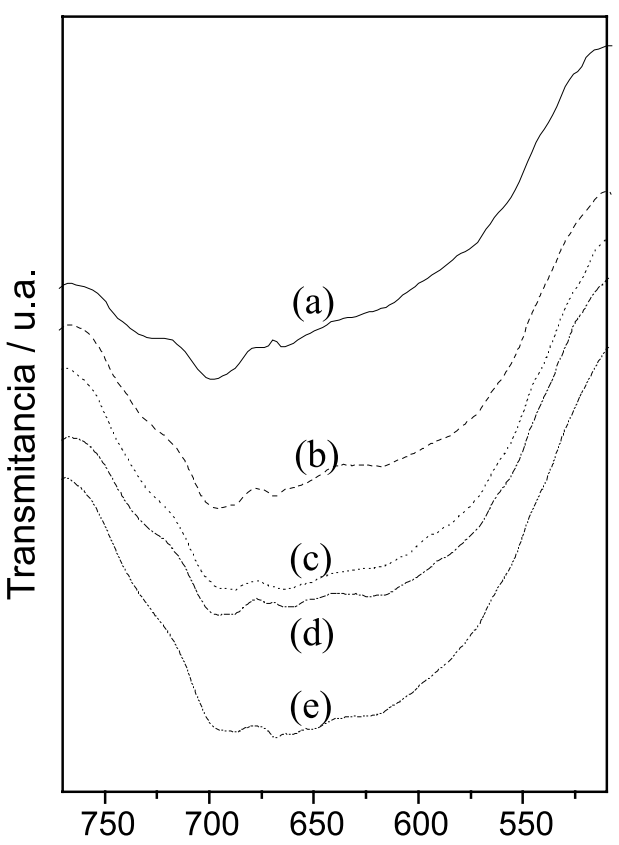

Número de Ondas $/ \mathrm{cm}^{-1}$

Figura 3. Espectros infrarrojos de los ormosiles con distintas concentraciones de TBT en el momento previo a la gelificación. (a) sin TBT, y con (b) $1 \%$, (c) $3 \%$, (d) $5 \%$, (e) $7 \%$ de TBT.

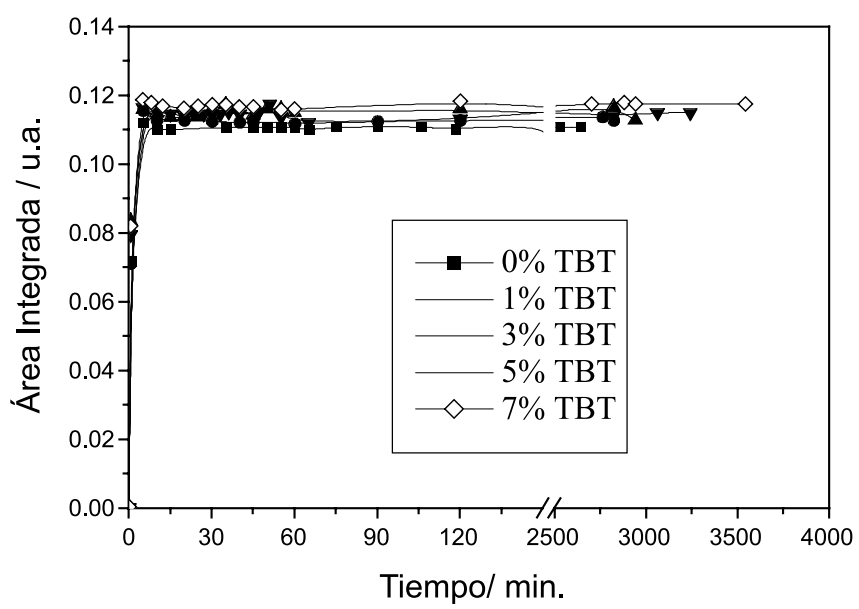

Figura 4. Evolución del área integrada correspondiente a la banda situada a $880 \mathrm{~cm}^{-1}$ para todas las muestras estudiadas con diferentes concentraciones de TBT.

cula de TEOS se ha seguido mediante la formación de etanol, ya que es la única molécula que puede dar lugar a dicho alcohol. La molécula de etanol presenta una banda muy intensa situada a $881 \mathrm{~cm}^{-1}$ correspondiente a la vibración de esqueleto de la molécula, la cual es perfectamente distinguible de las que presentan el resto de alcoholes. En la Figura 4 se muestra la evolución de la formación de etanol, y en ella puede verse como éste es generado en los cinco primeros minutos, resultado que concuerda perfectamente con el de Iwamoto y col. (14) quienes demostraron, mediante RMN, que se requerían un máximo de 5 minutos para la hidrólisis del TEOS en una disolución que contuviera PDMS. Puede verse también en esta Figura 4 como después de dicho tiempo el área integrada es constante con el tiempo de reacción así como que también es prácticamente independiente de la concentración de TBT que se adicione al sistema, lo que indica que todo el TEOS se ha hidrolizado en dichos 5 minutos de reacción. 
La hidrólisis del TBT se ha seguido mediante la banda situada a $1130 \mathrm{~cm}^{-1}$ y que es asignada por distintos investigadores a los enlaces Ti-O-C de la molécula de TBT $(23,24)$. En la Figura 5 está representada la evolución de dicha banda pudiéndose ver como aumenta durante la etapa de adición del TBT a la disolución, y una vez finalizada dicha etapa la banda disminuye hasta casi desaparecer en los momentos finales próximos a la gelificación. Esta evolución se debe interpretar de la siguiente forma: el aumento que tiene lugar de la banda del TBT durante la etapa de adición de éste se debe a que en la disolución no existe bastante agua para que se de la hidrólisis completa, pues todo el agua se ha empleado en hidrolizar al TEOS, si bien como el TBT es una molécula fácilmente hidrolizable en presencia de agua, una vez que ésta es generada por las reacciones de autocondensación o de policondensación TEOS-PDMS, se empleará en hidrolizar al TBT haciendo decrecer el área de la banda originada por dicha molécula. El hecho de que una vez que exista bastante cantidad de agua en el medio no todo el TBT se hidrolice (la banda no desaparece ni en los momentos finales próximos a la gelificación) se debe a que es muy difícil hidrolizar todos los radicales alcóxido de lo molécula de TBT tal y como han puesto de manifiesto numerosas investigaciones $(23,25-27)$.

La hidrólisis del TEOS origina grupos silanol (Si-OH) los cuales participan en las reacciones de autocondensación o de copolimerización (reacciones 2 y 3) mostradas anteriormente. En varias investigaciones se ha demostrado que la espectroscopia IR es capaz de diferenciar la formación de redes tridimensionales o lineales formadas por enlaces $\mathrm{Si}$ O-Si tras la autocondensación de los grupos silanol. Dichas redes o estructuras dan lugar a dos bandas situadas a 1180 y $1150 \mathrm{~cm}^{-1}$, respectivamente. La presencia de dichas bandas se ha demostrado en diversos trabajos gracias al proceso de deconvolución espectral tal y como el seguido en este trabajo $(28,29)$. En la Figura 6 se muestra la evolución de estas dos bandas, pudiéndose observar como las estructuras tridimensionales aumentan rápidamente durante los primeros 30-40 minutos de reacción, es decir durante la etapa de adición. A partir de dicho tiempo estas estructuras muestran un comportamiento diferente, así si la disolución no contiene TBT dichas estructuras tienden a decrecer, pero si en la disolución existe TBT entonces crecen ligeramente, a la vez que el incremento es mayor cuanto mayor es el contenido en TBT.

Por lo que respecta a las estructuras lineales de Si-O-Si (representadas en la Figura 6b), éstas crecen más deprisa que las tridimensionales alcanzando su máxima concentración a los 25 minutos de reacción. Pasado este tiempo el comportamiento depende de la concentración de TBT en el medio, así cuando no se adiciona TBT dichas estructuras presentan un descenso continuo hasta que la muestra gelifica, pero si la muestra contiene TBT el descenso es solo hasta los 100-120 minutos y luego permanecen constantes.

En la Figura 6, tanto la 6a como la 6b, puede comprobarse como las áreas integradas de las estructuras lineales y tridimensionales son más elevadas para la muestra que no contiene TBT, lo que indica que la adición de TBT desfavorece la formación de dichas estructuras probablemente debido a que se forman enlaces Ti-O-Si en los que el átomo de Ti pertenece a una nanopartícula de hidróxido de titanio hidratada, tal y como más tarde se comentará.

De acuerdo con la reacción [3] mostrada anteriormente los grupos $\mathrm{Si}-\mathrm{OH}$ copolimerizan con las moléculas de PDMS

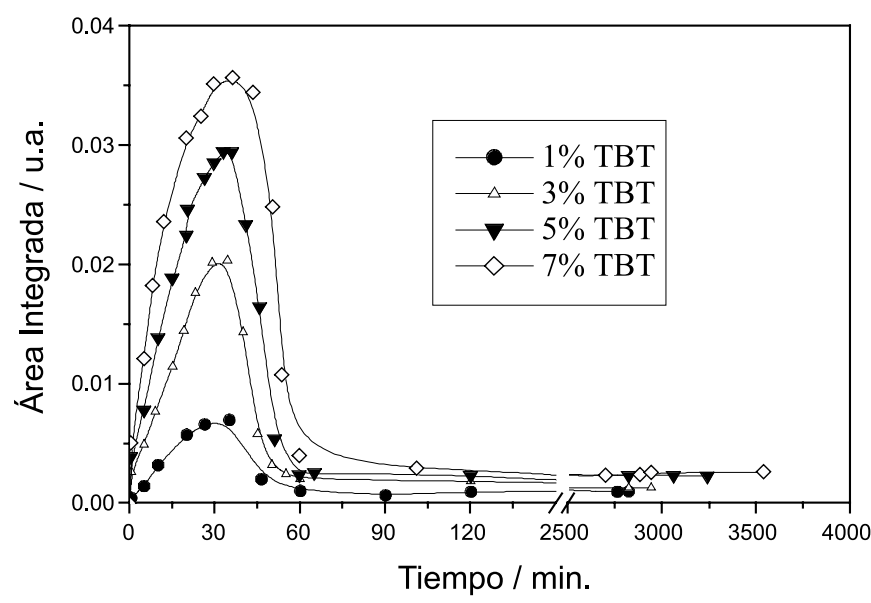

Figura 5. Evolución del área integrada de la banda situada a $1130 \mathrm{~cm}^{-1}$ para todas las muestras estudiadas con diferentes concentraciones de TBT.

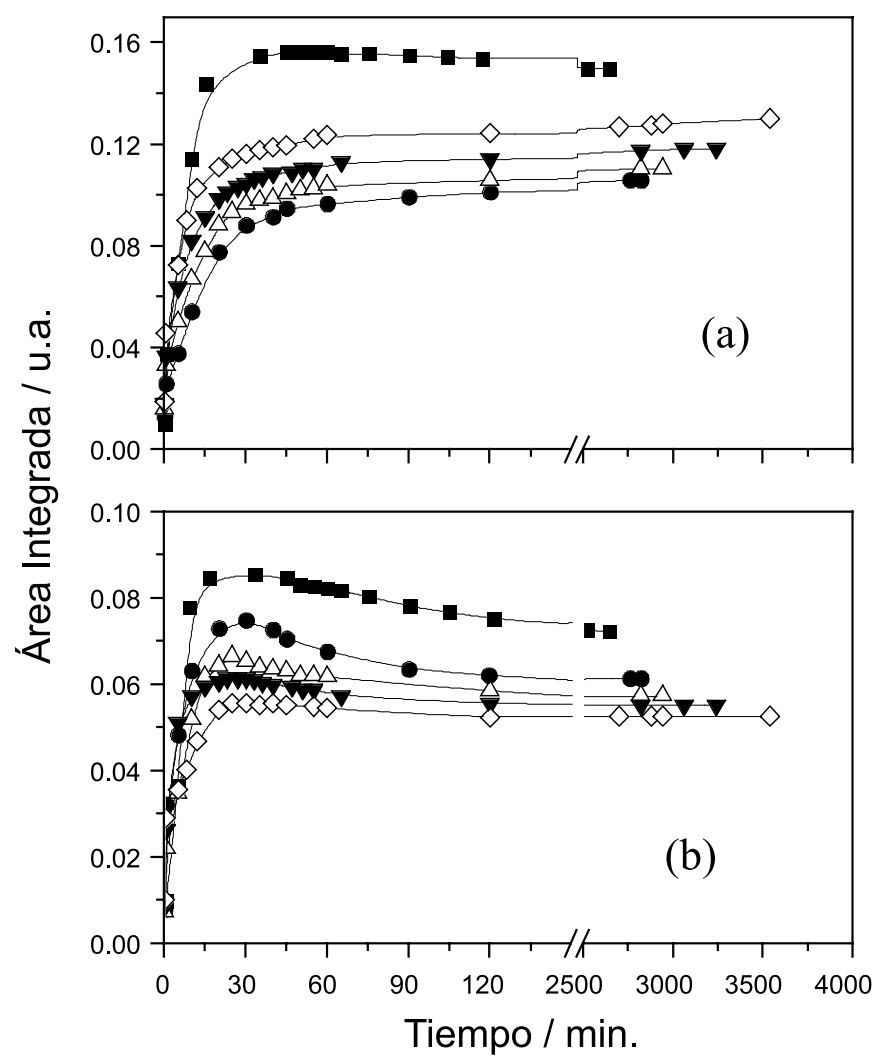

Figura 6. Evolución de las áreas integradas correspondientes a las bandas localizadas en (a) $1180 \mathrm{~cm}^{-1}$ y en (b) $1158 \mathrm{~cm}^{-1}$, para todas las muestras estudiadas en función de la concentración de TBT. - $\mathbf{-}$ - sin

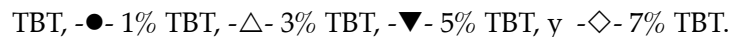

y dan lugar a una banda situada a $850 \mathrm{~cm}^{-1}$. Esta banda se ha representado en la Figura 7 en donde se puede comprobar como dicha reacción comienza en los primeros momentos, es decir nada más hidrolizarse el TEOS y que continúa creciendo hasta el minuto 80 aproximadamente, momento a partir del cual ya el crecimiento es menor hasta que la muestra gelifica. Esta reacción de copolimerización es dependiente de la concentración de TBT en la disolución, decreciendo dicha 
velocidad cuando la concentración de TBT aumenta, resultado similar al encontrado por Babonneau y col. $(6,30)$ los cuales mostraron que los alcóxidos de titanio pueden actuar como agentes de polimerización o como catalizadores de condensación de moléculas de PDMS. Por otro lado si se comparan las Figuras 6 ( $\mathrm{a}$ y b) y 7 , se puede concluir que las reacciones de autocondensación predominan frente a las de copolimerización, resultado que está de acuerdo con el descrito por Iwamoto y col. (14).

Por otro lado la reacción de copolimerización también se puede dar entre grupos $\mathrm{Si}-\mathrm{OH}$ y $\mathrm{Ti}-\mathrm{OH}$ para formar enlaces Si-O-Ti, los cuales dan una banda sobre los $936 \mathrm{~cm}^{-1}(12,31-$ 33). La evolución de esta banda se muestra en la Figura 8. Aquí hay que aclarar que no es posible distinguir si los grupos Ti-OH se han unido a grupos Si-OH del TEOS hidrolizado o a grupos Si-OH de final de cadena del PDMS, hecho que si es posible cuando se utilizan alcóxidos de Boro (34). En esta Figura 8 se observa que los enlaces Si-O-Ti aumentan durante la etapa de adición del TBT e incluso hasta los minutos 60-80 de reacción. A partir de dichos tiempos estos enlaces permanecen prácticamente constantes. Se puede observar como al aumentar la concentración de TBT aumenta el área integrada de dicha banda, es decir que se forman más enlaces Si-O-Ti cuanto mayor es el contenido en alcóxido de titanio en la reacción. El hecho de que la banda de $936 \mathrm{~cm}^{-1}$ no desaparezca en el transcurso de la reacción indica que estos enlaces se mantienen durante todo el tiempo, resultado que es similar al encontrado por Babonneau $(6,18)$ y Kotani y col. $(33)$, los cuales mostraron que dichos enlaces se forman durante la etapa de hidrólisis, luego se mantienen en el gel, pero desaparecen cuando éste envejece y se seca.

Como se ha comentado anteriormente la hidrólisis del TBT da lugar a grupos Ti-OH los cuales autocondensan formando enlaces Ti-O-Ti. Sánchez y col $(35,36)$ mostraron que cuando se utiliza un medio ácido para esta reacción los iones $\mathrm{H}^{+}$ actúan como inhibidores de la reacción de autocondensación y que el estado final del sistema depende fundamentalmente de la relación $\mathrm{H}^{+}$/ TBT más que de la concentración de $\mathrm{H}^{+}$. Si la relación molar $\mathrm{H}^{+}$/ TBT es menor de 0.005 , entonces siempre tiene lugar la precipitación, pero si dicha relación es mayor de 0.005 entonces se pueden obtener geles o soles (estos últimos si dicha relación es alta). Las relaciones molares utilizadas en el presente trabajo oscilan entre 1,6 y 11,2 para las diferentes concentraciones de TBT, por lo que de acuerdo con Sánchez y col. $(35,36)$, los tiempos de gelificación deberían ser superiores a 1 año si en el sistema no existiera TEOS ni PDMS. Sin embargo la gelificación de todas las muestras estudiadas indica que aquella es dependiente fundamentalmente de la concentración de TEOS, PDMS, $\mathrm{H}_{2} \mathrm{O}$ y $\mathrm{HCl}$, si bien el TBT debe también influir. Así se observó que los tiempos de gelificación variaron desde 44 a 59 horas para las muestras que no tenían TBT a la que contenía el $7 \%$, luego el TBT retarda la gelificación debido a la alta concentración de $\mathrm{H}^{+}$en el medio. Por otro lado y de acuerdo con Sánchez y col $(35,36)$, las elevadas relaciones $\mathrm{H}^{+} / \mathrm{TBT}$ empleadas indican que la precipitación de hidróxido de titanio está impedida, de ahí que los materiales obtenidos sean transparentes tal y como se indicó al principio.

Por otro lado, a pesar de dicha elevada concentración de $\mathrm{H}^{+}$, es posible que autocondensen los grupos Ti-OH formando nanopartículas de hidróxido de titanio hidratado tal y como propuso Babonneau (4). La formación de estas partículas puede ser determinada mediante espectroscopia IR analizan-

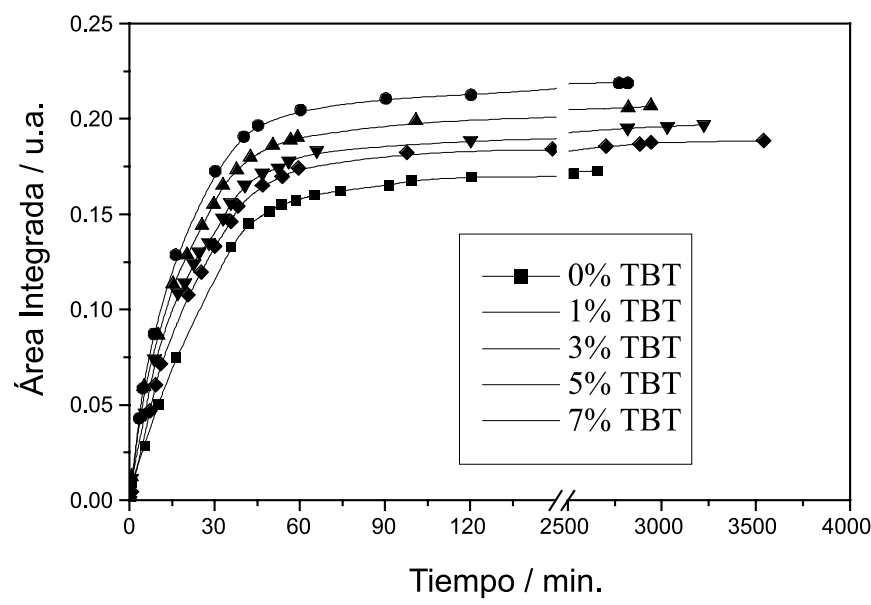

Figura 7. Evolución del área integrada correspondiente a la banda situada a $850 \mathrm{~cm}^{-1}$ para todas las muestras estudiadas y para diferentes concentraciones de TBT.

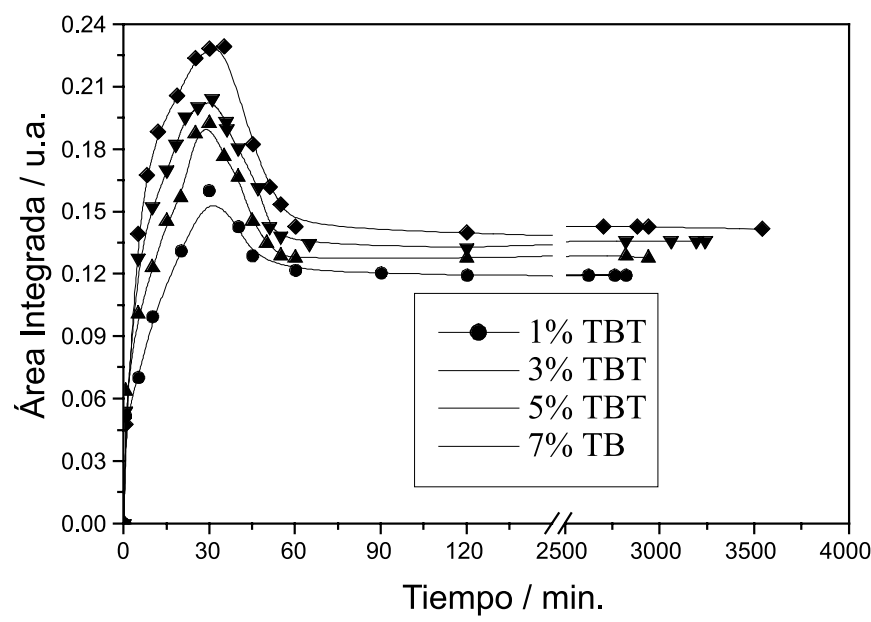

Figura 8. Evolución del área integrada de la banda situada a $936 \mathrm{~cm}^{-1}$ para todas las muestras estudiadas y para diferentes concentraciones de TBT.

do las amplias bandas que originan los enlaces Ti-O-Ti sobre los $500 \mathrm{~cm}^{-1}$. Así y tal y como se comentó anteriormente sobre la Figura 3 en ella se observó que la absorbancia de la región espectral 770-500 $\mathrm{cm}^{-1}$ aumentaba con el contenido de TBT adicionado, luego dicho aumento debe estar relacionado con la formación de los citados Ti-O-Ti enlaces y dicho aumento puede servir para analizar su evolución en la reacción. Sin embargo para llevar a cabo tal análisis se debe tener en cuenta que en dicha región espectral también aparecen las bandas de flexión fuera del plano de los enlaces $\mathrm{O}-\mathrm{H}$ de las moléculas de $\mathrm{H}_{2} \mathrm{O}$ y de alcoholes, siendo dichas bandas muy anchas aunque poco intensas. Teniendo en cuenta este hecho, se ha procedido a realizar la deconvolución de la zona espectral 750-500 $\mathrm{cm}^{-1}$ y se ha determinado el área integrada de las bandas que la componen. La elección de esta zona de $750-500 \mathrm{~cm}^{-1}$ no ha sido aleatoria sino que primeramente se ha hidrolizado una mezcla de TBT e isopropanol con 4 moles de $\mathrm{H}_{2} \mathrm{O}$, se ha obtenido un precipitado de hidróxido de titanio el cual se ha filtrado y secado y posteriormente se ha analizado por FT-IR observándose que es en dicha región espectral donde aparecen sus bandas más intensas, de ahí que sea esta región la que se utilice en el análisis de las reacciones estudiadas en este trabajo. En la Figura 9 se muestra la evolución de dicha área total de la región $750-500 \mathrm{~cm}^{-1}$. 
Para hacer un análisis más exacto de los resultados obtenidos en el estudio de la zona espectral $750-500 \mathrm{~cm}^{-1}$, se debe asumir que:

a) todas las reacciones consumen la misma cantidad de moléculas de $\mathrm{H}_{2} \mathrm{O}$ (lo que en principio es cierto),

b) la cantidad de moléculas de $\mathrm{H}_{2} \mathrm{O}$ generadas por las reacciones de condensación de grupos $\mathrm{Si}-\mathrm{OH}$ es similar para todos los casos, $\mathrm{y}$

c) como la cantidad de alcohol (iPrOH) es la misma en todas las reacciones, entonces el aumento del área integrada observado en esta Figura 9 se tiene que deber solamente a dos factores: primero, al etanol formado por la hidrólisis del TEOS y, segundo, a la formación de enlaces Ti-O-Ti. Como la cantidad de etanol depende de la de TEOS, entonces la mayor cantidad de etanol formada será para la muestra que no contienen TBT, por lo tanto cualquier diferencia que se haga entre las áreas integradas de dicha zona espectral para cualquier muestra que contenta TBT con respecto a la que no contiene TBT, se debe obtener un valor que será positivo o negativo en función solamente de la cantidad de enlaces Ti-O-Ti formados.

En la Figura 9 la curva correspondiente a la muestra que no contiene TBT presenta un aumento que se tiene que deber a la formación de etanol y de agua, tal y como se acaba de comentar. Sin embargo para el resto de las muestras los aumentos observados serán debidos a la presencia de agua, de etanol y de los posibles enlaces Ti-O-Ti. Como en dicha Figura 9 se observa que para todas las muestras que contienen TBT las áreas integradas son superiores a la de la muestra que no contiene TBT, eso indica que en todas ellas se forman enlaces Ti-O-Ti. A su vez se observa que a medida que aumenta la concentración de TBT en el medio mayor es el área integrada de la región espectral analizada, lo que de nuevo corrobora la formación de dichos enlaces. Esta formación tiene lugar durante los primeros 60 minutos de reacción (de acuerdo con la Figura 9), es decir durante los 35 minutos de adición del TBT y los 25 minutos posteriores. A partir del minuto 60 el área integrada de dicha zona aumenta ligeramente, indicando que la formación de más enlaces Ti-O-Ti es ya muy lenta. Según este resultado es posible que se formen nanopartículas de hidróxido de titanio hidratado, si bien la concentración de éstas debe ser pequeña ya que todos los materiales obtenidos una vez gelificados fueron completamente transparentes.

Finalmente solo comentar que como se había dicho en la Introducción el PDMS es un polímero que presenta estructuras cíclicas y lineales en un equilibrio que depende del medio en el que esté. Estas estructuras dan lugar a dos bandas situadas a 813 y $800 \mathrm{~cm}^{-1}$, para las estructuras cíclicas y para las lineales, respectivamente, siendo las dos responsables de la intensa banda que de el PDMS sobre los $805 \mathrm{~cm}^{-1}$, y que está formada por las dos anteriores. Estas dos bandas se han deconvolucionado, representándose en la Figura 10 la evolución de ambas. Así se puede ver como las estructuras cíclicas aumenta rápidamente nada mas adicionar la disolución hidrolizante, a la vez que las estructuras lineales muestran el comportamiento contrario. Sin embargo para tiempos ya próximos a la gelificacion las estructuras cíclicas mantienen el área integrada mientras que las lineales decrecen ligeramente lo que indica que son éstas las que se están incorporando a la estructura del híbrido, resultado similar al encontrado por Iwamoto y col. (14).

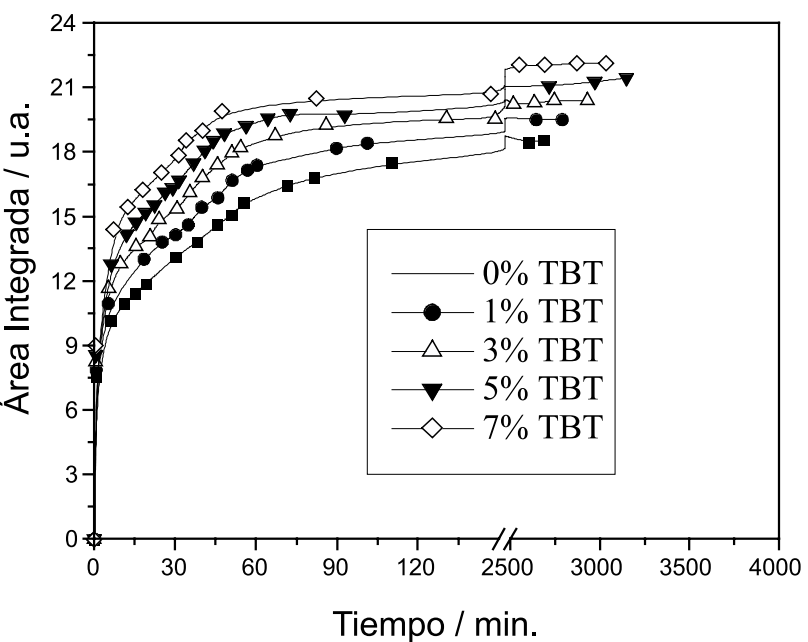

Figura 9. Evolución del área integrada de la zona espectral situada entre 750 y $500 \mathrm{~cm}^{-1}$ para todas las muestras estudiadas y para diferentes concentraciones de TBT.

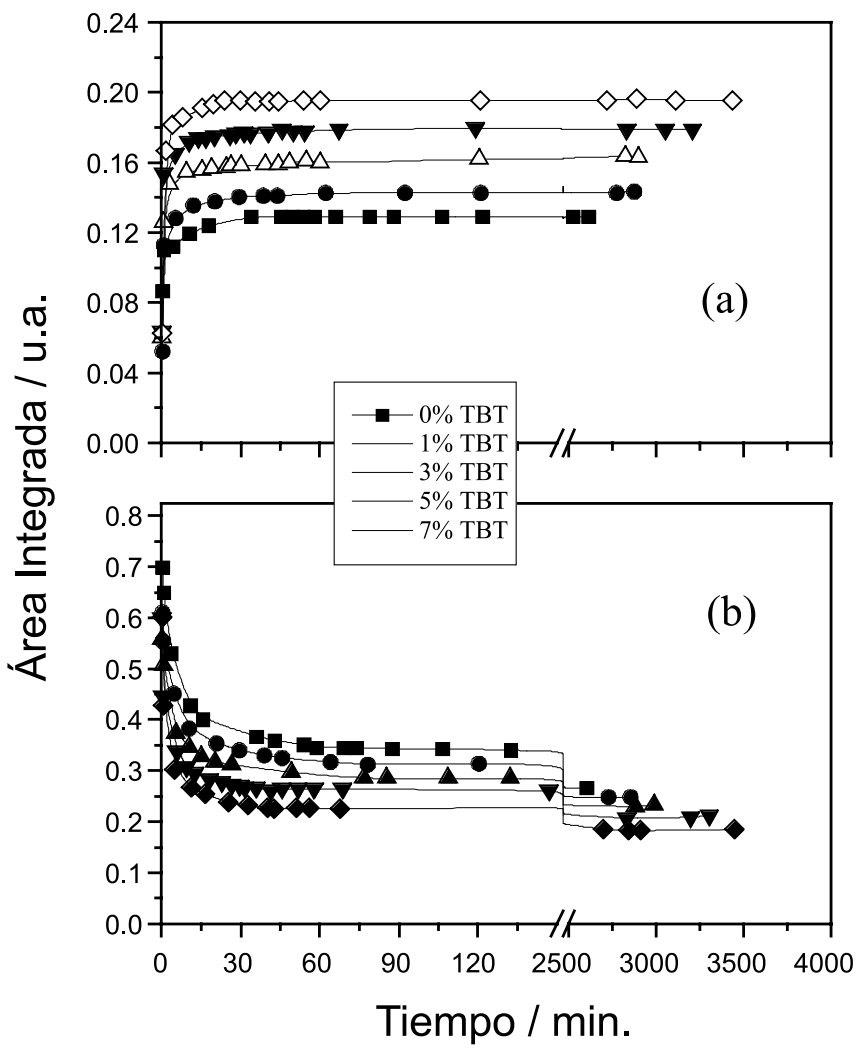

Figura 10. Evolución de las áreas integradas de las bandas situadas a (a) $813 \mathrm{~cm}^{-1}$ y (b) $800 \mathrm{~cm}^{-1}$ para todas las muestras estudiadas con

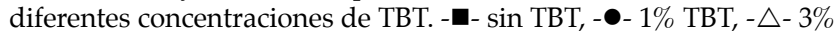
TBT, $-\nabla-5 \%$ TBT, y $-\diamond-7 \%$ TBT.

\section{CONCLUSIONES}

De acuerdo con lo que se acaba de comentar las conclusiones de este trabajo se pueden resumir como sigue. La hidrólisis del TEOS es muy rápida y transcurre en los primeros 5 minutos de reacción; en estos minutos tiene lugar la formación de estructuras tridimensionales y lineales de enlaces Si-O-Si a partir de la autocondensación de grupos silanol; también en estos primeros momentos comienzan a policondensar las moléculas de TEOS hidrolizado (los grupos silanol, en con- 
creto) con las moléculas de PDMS. Si el agua adicionada se empleaba para hidrolizar al TEOS, ahora todas estas reacciones dan origen a moléculas de agua que sirven para hidrolizar al TBT que se añade de forma continua. El TBT hidrolizado genera grupos Ti-OH que autocondensan para formar enlaces $\mathrm{Ti}-\mathrm{O}-\mathrm{Ti}$ o bien policondensan para formar enlaces Ti-O-Si, habiéndose encontrado que los enlaces Ti-O-Ti crecen durante todo el transcurso de la reacción, mientras que los enlaces Ti-O-Si crecen mientras se adiciona TBT al medio pero luego decrecen lo que indica que se rompen debido a su baja estabilidad en el medio utilizado. Se ha observado que la presencia de TBT desfavorece la formación de estructuras tridimensionales o lineales de enlaces Si-O-Si así como también desfavorece la copolimerización de grupos $\mathrm{Si}-\mathrm{OH}$ con moléculas de PDMS, un hecho que está de acuerdo con el aumento de los tiempos de gelificación cuando aumenta la concentración de TBT en el medio. Finalmente decir que la presencia de estructuras cíclicas y lineales de PDMS se ve alterada por el medio de reacción, siendo las lineales las que se incorporan a la estructura del híbrido.

\section{AGRADECIMIENTOS.}

Este trabajo ha sido realizado de acuerdo a un Proyecto de Investigación del MCYT correspondiente al Plan Nacional y cuya referencia es MAT2002-03891. Lucía Téllez quiere agradecer al Instituto Politécnico Nacional y al Consejo Nacional de Ciencia y Tecnología de México (CONACyT) la ayuda prestada de acuerdo con la beca de referencia 72432.

\section{BIBLIGRAFÍA}

1. Lisa C. Klein. Sol-gel technology for thin films, fibers, performs, electronics and specialty shapes (Noyes Publications, New Jersey, U.S.A, 1988).

2.D.R. Uhlmann, G. Teowee. "Sol-gel science and technology: current state and future prospects". J. Sol-Gel Sci. Technol. 13 (1/2/3) 153-162 (1998).

3.H. Schmith and G. J. Philipp. "New materials for contact lenses prepared from $\mathrm{Si}$ and Ti-alkoxides by the sol-gel process". J. Non-Cryst. Solids 63, 283-292 (1984)

4.C. Sanchez and F. Ribot. "Design of hybrid organic-inorganic materials synthesized via sol-gel chemistry". New J. Chem. 18, 1007-1047 (1994).

5.H. Schmidt. "New type of non-crystalline solids between inorganic and organic materials". J. Non-Cryst. Solids, 73, 681-691 (1985).

6.F. Babonneau, "Hybrid Siloxane-Oxide Materials Via Sol-Gel Processing: Structural Characterization". Polyhedron, 13, (8) 1123-1130 (1994).

7.B. E. Yoldas. "Introduction and Effect of Structural Variations in Inorganic Polymers and Glass Networks". J. Non-Cryst. Solids, 51, 105-121 (1982).

8.Y. Hu, J. D. Mackenzie. "Structure-related mechanical properties of Ormosils by sol-gel process". Mater. Res. Soc. Symp. Proc. 271, 681-685 (1992).

9.G. H. Floch, P. F. Belleville, J. J. Priotton, P.M. Pergon, C. S. Dojonneau and J. Guerain. "Sol-gel optical coatings for lasers. I". Am. Ceram. Soc. Bull. 1 (10) 60-65 (1995).

10.J. D. Mackenzie, Y. J. Chung and Y. Hu. "Rubbery Ormosils and their Applications". J. Non-Cryst. Solids. 147/148, 271-279 (1992).

11.S. Diré, F. Babonneau, C. Sánchez, J. Livage. Sol-Gel synthesis of siloxane-oxide hybrid coatings. J. Mater. Chem. 2 (2), 239-244 (1992).
12.S. Katayama, I. Yoshinaga and N. Yamada. "Synthesis of inorganic-organic hybrids from metal alkoxides and silanol-terminated polydimethylsiloxane". Mater. Res. Soc. Symp. Proc. 435, 321-326 (1996).

13.C. S. Parkust, W. F. Doyle, L. A. Silverman, S. Singh, M. P. Andersen, D. McClurg, G. E. Wnek and D. R. Uhlmann. “Siloxane Modified $\mathrm{SiO}_{2}-\mathrm{TiO}_{2}$ Glasses Via Sol-Gel”. Mater. Res. Soc. Symp. Proc. 73, 769-773 (1986).

14.T. Iwamoto, K. Morita, J. D. Mackenzie. "Liquid state ${ }^{29} \mathrm{Si}$ NMR study on the sol-gel reaction mechanisms of ormosils". J. Non-Cryst. Solids 159 (1-2), 6572 (1993)

15.R.H. Glaser and G.L. Wilkes "Structure Property Behavior of Polydimethylsiloxane and Poly(tetramethylene Oxide) Modified TEOS Based Sol-Gel Materials. V. Effect of Titaniumisopropoxide incorporation". Polym. Bull. 19, 51-57 (1988).

16.F. Rubio, J. Rubio and J.L. Oteo. "Effect on the Pore Structure of $\mathrm{SiO}_{2}$-PDMS Ormosils". J. Sol-Gel Sci. Technol. 18, 105-113 (2000).

17.T. Iwamoto and J.D. Mackenzie. "Hard Ormosils Prepared with Ultrasonic Irradiation". J. Sol-Gel Sci. Technol. 4, 141-150 (1995).

18.F. Babonneau. ${ }^{29} \mathrm{Si},{ }^{17} \mathrm{O}$ Liquid NMR and ${ }^{29} \mathrm{Si}$ CP-MAS NMR characterization of Siloxane-Oxide Materials, $\left[\left(\mathrm{CH}_{3}\right)_{2} \mathrm{SiO} / \mathrm{ZrO}_{2}\right]$ ". Mat. Res. Soc. Symp. Proc. 346, 949-960 (1994).

19.S. Diré, F. Babonneau, G. Carturan, J. Livage. “Synthesis and Characterization of Siloxane-Titania Materials". J. Non-Cryst. Solids 147/148, 62-66 (1992).

20.L.J. Bellamy. "Organo-Silicon Compounds", pp. 374-384 en The Infrared Spectra of Complex Molecules. Chapman and Hall, London, 1975.

21.D. Vivien, J. Livage and C. Mazieres. "Nature des Précipités d'oxydes hydratés des métaux du groupe IV A. I. Analyse thermique et spectroscopie infrarouge." J. Chim. Phys. 67, 199-204 (1970).

22.M. J. Velasco, F. Rubio, J. Rubio and J. L. Oteo. "DSC and FT-IR Analysis of the Drying Process of Titanium Alkoxide Derived Precipitates". Thermochimica Acta 326, 91-97 (1999).

23.M. J. Velasco, F. Rubio, J. Rubio and J. L. Oteo. “Hydrolysis of Titanium Tetrabutoxide. Study by FT-IR Spectroscopy". Spectroscopy Letters 32 [2] 289-304 (1999).

24.S. Doeuff, M. Henry, C. Sanchez and J. Livage. "Hydrolysis of Titanium Alkoxides: Modification of the Molecular Precursor by Acetic Acid". J. NonCryst. Solids 89, 206-216 (1987).

25.F. Rubio, J. Rubio, P. Duran and J. L. Oteo. "Preparation of Nanometric Titanium Hydrous Oxied Particles by Vapour Phase Hydrolysis of Titanium Tetrabutoxide". J. Mater. Sci. 34, 3397-3404 (1999).

26.B. Yoldas. "Hydrolysis of Titanium Alkoxide and Effects of Hydrolytic Polycondensation Parameters". J. Mater. Sci. 21, 1087-1092 (1986).

27.A. Larbot, I. Laaziz, J. Marignan and J. F. Quinsonand. "Porous texture of a titanium oxide gel: evolution as a function of medium used". J. Non-Cryst. Solids 147/148, 157-165 (1992).

28.N. Viart and J.L. Rehspringer. "Study of the Action of Formamide on the Evolution of a Sol by $\mathrm{pH}$ Measurements and Fourier Transformed Infra-red Spectroscopy". J. Non-Cryst. Solids 195, 223-231 (1996).

29.F. Rubio, J. Rubio and J. L. Oteo. "Study of the Hydrolysis of Tetraethylortosilicate (TEOS)".Spectroscopy Letters 31 [1] 199-219 (1998).

30.F. Babonneau, L. Bois, J. Livage and S. Dire. "Structural investigation of solgel-derived hybrid siloxane-oxide materials using silicon- ${ }^{29}$ MAS-NMR spectroscopy". Mater. Res. Soc. Symp. Proc. 286, 289-294 (1993).

31.K.A. Andrianov. "Polymers with Inorganic Primary Molecular Chains". J. Polym. Sci. 52, 257-276 (1961).

32.I. Hasegawa, T. Nakamura and M. Kajiwara. Synthesis of Continuous Silicon Carbide-Titanium Carbide hybrid Fibers Through Sol-Gel Processing". Mater. Res. Bull. 31 [7] 869-875 (1996).

33.Y. Kotani, A. Matsuda, M. Tatsumisago and T. Minami. "Formantion of Anatase Nanocrystals in Sol-Gel Derived $\mathrm{TiO}_{2}-\mathrm{SiO}_{2}$ Thin Films with Hot Water Treatment". J. Sol-Gel Sci. Technol. 19, 585-588 (2000).

34.R. Peña-Alonso, J. Rubio, F. Rubio, J. L. Oteo. "FT-IR and Porosity Study of SiB-C-O Materials Obtained from TEOS-TEB-PDMS Derived Gel Precursors". J. Sol-Gel Sci. Tech. 26 (2003) 195 - 199

35.M. Kallala, C. Sánchez and B. Cabane. "SAXS Study of Gelation and Precipitation in Titanium-Based Systems". J. Non-Cryst. Solids 147 \& 148, 189-193 (1992).

36.M. Kallala, C. Sánchez and B. Cabane "Structures of Inorganic Polymers in Sol-Gel Processes Based on Titanium Oxide". Physical Review E. 48 [5] 36923704 (1993).

Recibido: 23.01.04

Aceptado: 15.04 .04 